\title{
Electron Holography at Low Voltages Exemplified by Graphene
}

\author{
Felix Börrnert, Axel Lubk, Falk Röder, Daniel Wolf, and Hannes Lichte \\ Institut für Strukturphysik, Technische Universität Dresden, 01062 Dresden, Germany
}

Low-voltage transmission electron microscopy (TEM) has re-emerged in the last decade with the advent of the hardware aberration correctors. While the early electron microscopes were operated at accelerating voltages regarded as low nowadays, the voltage was increased over the time in order to achieve the maximum possible resolving power essentially limited by the aberrations of the objective lens. However, the achievement of image resolution allowing characterization of crystal structures had to be paid with increased beam damage induced by the high energy of the imaging electrons. This restricts the investigation of whole classes of the materials containing light elements. The introduction of aberration correctors enables the investigation also of these materials at sufficient resolution with accelerating voltages of $80 \mathrm{kV}$ and even down to $20 \mathrm{kV}$ [1].

Transmission electron microscopy has several drawbacks resulting from the fact that it only records the squared amplitude of the image wave while losing most of the phase information. In particular, because of the phase contrast transfer function, one loses the information about extended structures, i.e., the exact projected electrostatic and magnetic field values encoded in the electron wave. Fortunately, the full wave information can be retrieved by means of off-axis electron holography [2].

Besides the fact that graphene is a material vividly investigated [3] it constitutes a perfect test specimen for TEM and electron holography because of its thickness of exactly one atom. In particular, this allows neglecting all multiple and dynamic scattering phenomena and identifying the projected potential of the object directly from the object exit wave. Moreover, graphene consists of carbon that is very light and thus approximates a pure phase object very well. On the other hand, graphene is susceptible to knock-on damage at normal TEM operation voltages, and hence the use of low acceleration voltages is mandatory [4].

In this contribution, using a FEI Tecnai F20 Cs-corr, we present an investigation about the benefits from using low acceleration voltages in off-axis electron holography. In electron holography the figure of merit of a hologram is the information content concatenating the maximum field of view, lateral resolution, and signal resolution [5]. The information content figure depends on the acceleration voltage directly and indirectly in several parameters like the improved sampling due to a larger maximum magnification, a stronger signal due to a larger interaction cross section and therefore shorter exposure times, a better stability of the biprism at lower filament voltages, and finally the superior characteristics (modulation transfer function and detection quantum efficiency) of the camera at $80 \mathrm{kV} \mathrm{[6].}$

Finally, we present the measurement of the electron phase shift by graphene and discuss the mean inner potential of graphene [7]. 
References:

[1] U Kaiser et al, Ultramicroscopy 111 (2011) p. 1239.

[2] H Lichte et al, Annual Review of Materials Research 37 (2007) p. 539.

[3] M H Rümmeli et al, Advanced Materials 23 (2011) p. 4471.

[4] J C Meyer et al, Physical Review Letters 108 (2012) p. 196102.

[5] H Lichte, Ultramicroscopy 108 (2008) p. 256.

[6] F Börrnert et al, Journal of Microscopy 249 (2013) p. 87.

[7] The research leading to these results has received funding from the European Union Seventh Framework Programme under Grant Agreement 312483 - ESTEEM2 (Integrated Infrastructure Initiative - I3). We thank P. Hartel and M. Haider for the alignment of the aberration corrector for $80 \mathrm{kV}$ operation, and S. Melkhanova for providing a graphene sample.
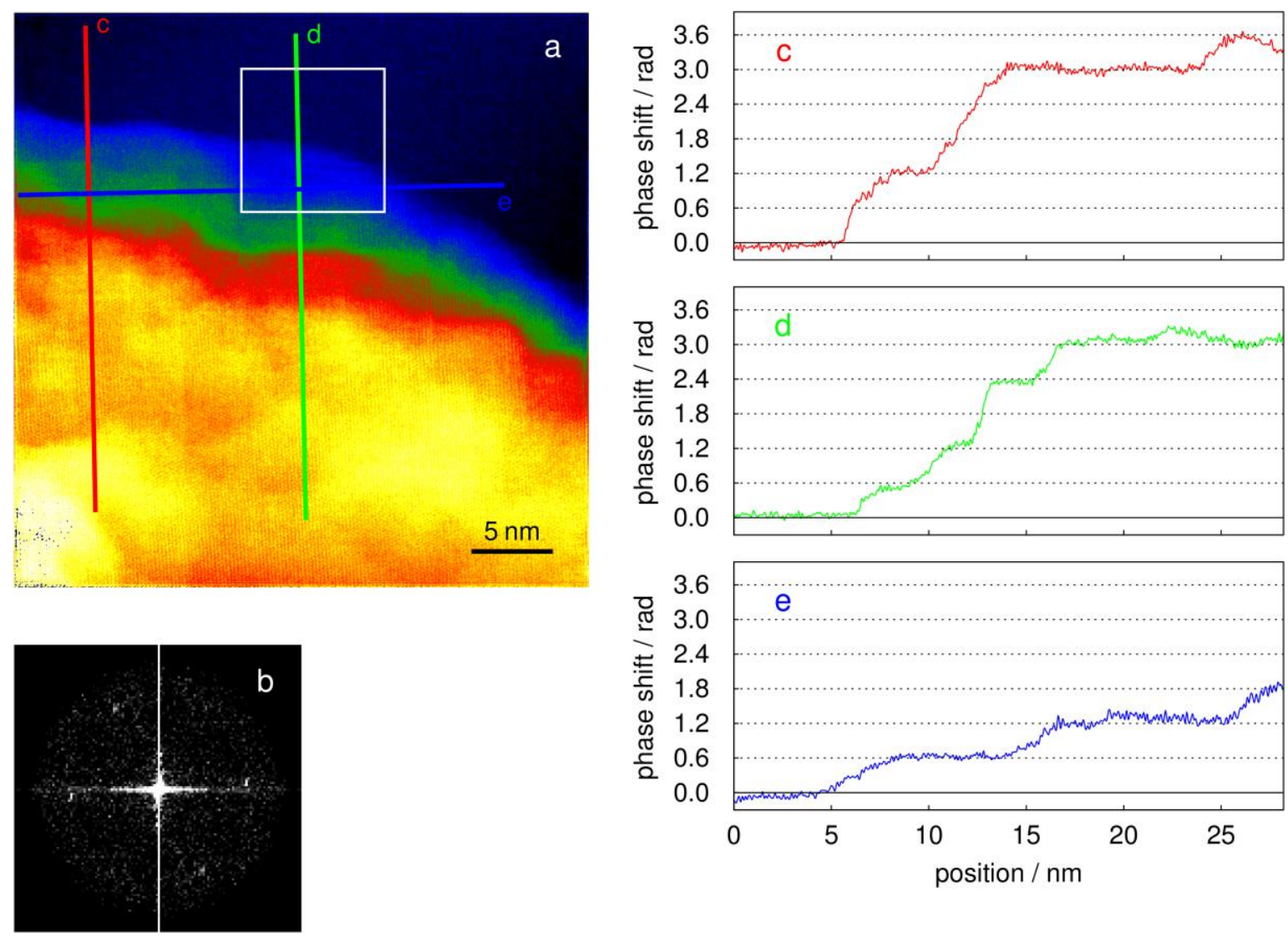

Figure 1. Electron holographic phase image of few-layer graphene. a) false color phase image showing the stacking of graphene layers; b) Fourier transform of the area indicated by the white box reveals corresponding diffraction spots; c), d, e) line profiles as indicated in a), revealing phase steps of 0.6 rad from single graphene layers. 